\title{
In-situ Transmission Electron Microscopy at Pressures Above 1 bar and Elevated Temperatures
}

Henny Zandbergen

Kavli Institute of Nanoscience, Delft University of Technology, Lorentzweg 1, 2628

CJ Delft, The Netherlands

Recent developments using MEMS techniques to make nanoreactors [1] allow in-situ TEM experiments at very high gas pressures and elevated temperatures. For instance we have succeeded in performing (de)hydrogenation studies of palladium with gas pressures up to 4.5 bar [2]. TEM imaging under these high pressures require a confinement of the gas to a small volume. In an environmental transmission electron microscope (ETEM) the gas is completely filling the sample holder area, resulting in a gas column of at least $10 \mathrm{~mm}$ high, and if one targets at high resolution information, the maximum gas pressure is roughly $10 \mathrm{mbar}$, depending of course also on the scattering potential of the gas molecules. In a MEMS nanoreactor the gas column length can be limited to roughly $10 \mu \mathrm{m}$, thus allowing a 1000 times larger pressure than an e-TEM and with a similar image resolution. To achieve such a short gas column the MEMS nanoreactor has to contain very thin membranes that are airtight. Evidently, the thinner the membranes the better for the image resolution, but if they are too thin they will not withstand the pressure difference.

Figure 1 shows the tip of a TEM holder fitted with a MEMS nanoreactor that we have developed. The MEMS-based nanoreactor contains a microheater, two electrontransparent windows and a gas inlet and outlet. The holder contains various O-rings to have leak-tight connections with the nanoreactor.

The system was tested with the (de)hydrogenation of $\mathrm{Pd}$ at pressures up to $4.5 \mathrm{bar}$. The Pd film consisted of islands being $15 \mathrm{~nm}$ thick and 50 to $500 \mathrm{~nm}$ wide. In electron diffraction mode we observed reproducibly a crystal lattice expansion and shrinkage owing to hydrogenation and dehydrogenation, respectively. In selected-area electron diffraction and bright/dark-field modes the (de)hydrogenation of individual $\mathrm{Pd}$ particles was followed. Some Pd islands are consistently hydrogenated faster than others. When thermally cycled, thermal hysteresis of about $10-16{ }^{\circ} \mathrm{C}$ between hydrogen absorption and desorption was observed for hydrogen pressures of 0.5 to 4.5 bar, in agreement with Siefert experiments on bulk samples.

Importantly, experiments at 0.8 bar and 3.2 bar with a wide range of electron beam intensities showed that the (de)hydrogenation temperature is not affected by the electron beam, showing that this is a reliable and fast method to investigate hydrogen storage materials providing information at the nanometer scale.

Evidently the in-situ instrumentation can also be used for TEM studies of other processes, like oxidation-reduction reactions of metallic and semiconducting particles, and corrosion of metal surfaces. Also it can be used in combination with other analysis techniques like infrared spectroscopy and scanning transmission X-ray microscopy. In the talk various examples will be given and the possibilities and limitations will be discussed.

\section{References}

[1] J.F. Creemer et al, Ultramicroscopy 108 (2008) 993.

[2] T. Yokosawa et al, Ultramicroscopy 112 (2012) 47. 


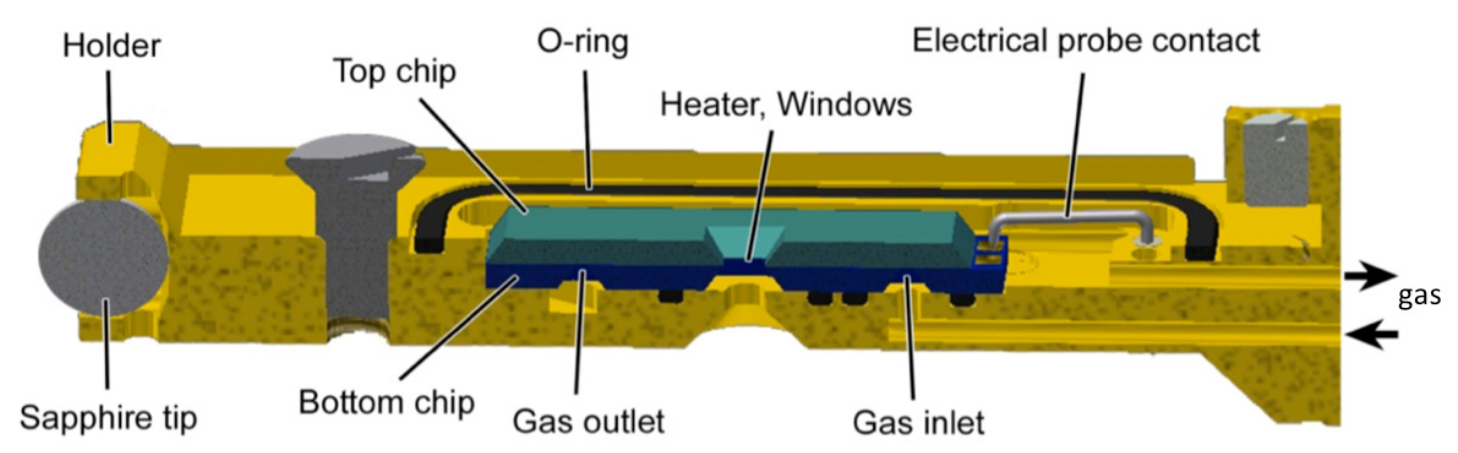

Fig. 1. Schematic diagram of the nanoreactor inserted in the sample holder.

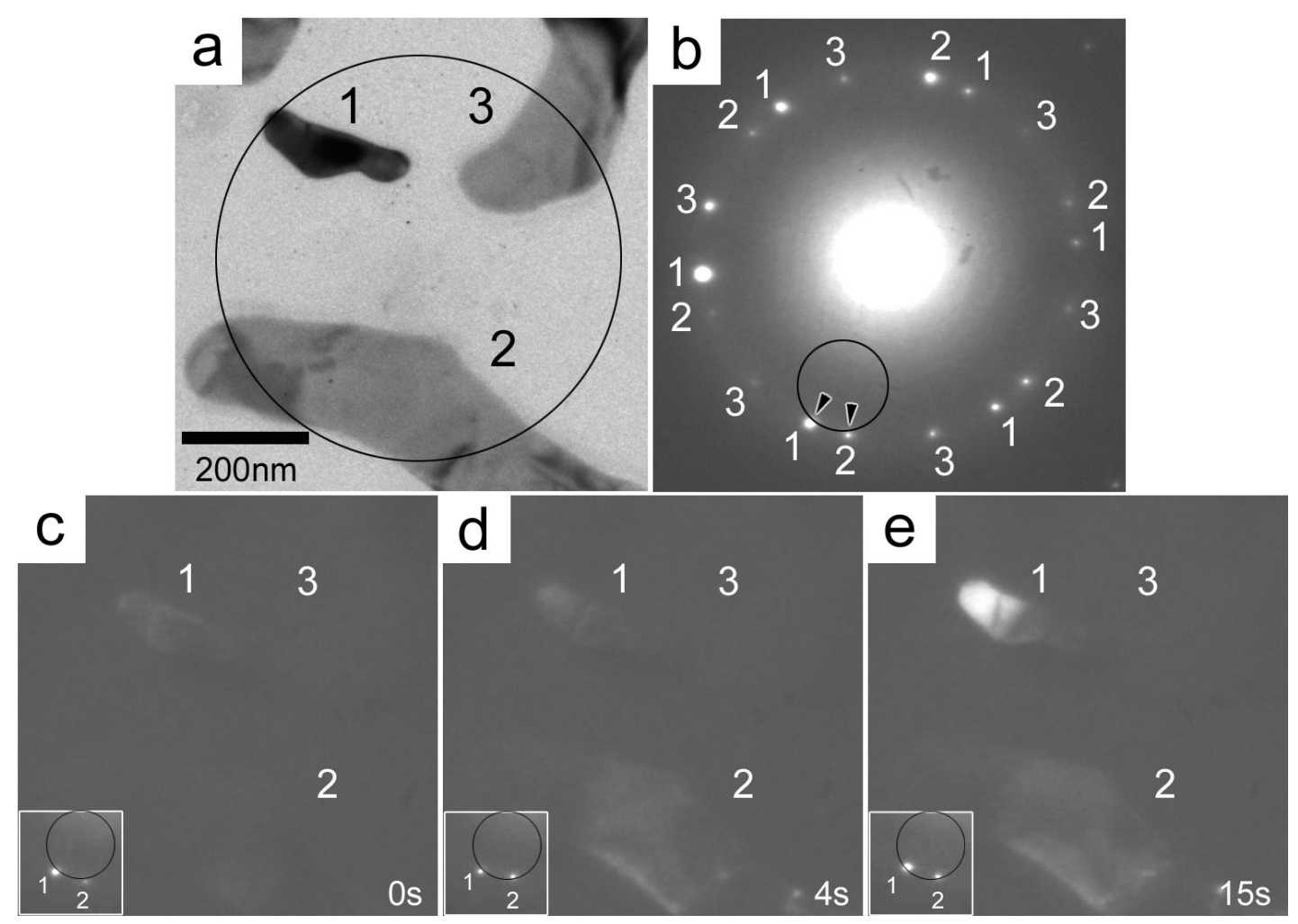

Fig. 2. (a) and (b) BF image and SAED pattern at 0.8 bar, respectively, where the SAED pattern was taken from the area in the black circle in (a). (c-e) Dark-field images taken $0 \mathrm{sec}, 4 \mathrm{sec}$ and $15 \mathrm{sec}$ after decreasing the temperature from $140^{\circ} \mathrm{C}$ to RT. The position of the objective aperture is indicated by the black circle in the SAED pattern. 